\title{
Optical design of laser beam shaping systems
}

\author{
David L. Shealy* \\ University of Alabama at Birmingham \\ Department of Physics, $15303^{\text {rd }}$ Avenue South, CH310 \\ Birmingham, AL 35294-1170 USA
}

\begin{abstract}
An overview of the history and current practices of laser beam shaping is presented. When diffraction effects are not important, geometrical methods for laser beam shaping (ray tracing, conservation of energy within a bundle of rays, and the constant optical path length condition) can be used to determine system configurations, including aspheric elements and spherical-surface GRIN lenses, which are required to change the intensity profile into a more useful form. Geometrical optics-based design methods are presented for shaping both rotationally and rectangular symmetric laser beam profiles. Applications of these techniques include design of a two-plano-aspheric lens system for shaping a rotationally symmetric Gaussian beam, a two-mirror system with no central obscuration for shaping an elliptical Gaussian input beam, and a three-element GRIN system for shaping a rotationally symmetric Gaussian beam.
\end{abstract}

Keywords: laser; beaming shaping; geometrical optics; optical design; irradiance mapping

\section{INTRODUCTION}

Optical design of laser beam shaping systems has evolved considerably from the early work of Frieden ${ }^{1}$ and Kreuzer $^{2}$ during the 1960's to the contemporary work of many summarized in Refs. 3, 4, and 5. This early work articulated well the goals of some contemporary laser beam shaping applications. Namely, Frieden and Kreuzer sought to define an optical system that would transform an input plane wave with a Gaussian irradiance profile into an output plane wave with uniform irradiance. Conservation of energy along a bundle of rays was used to establish a non-linear mapping of the ray coordinates between the input and output planes. Frieden shows that the phase of the beam over the output plane may vary by $20 \lambda$ after redistribution of the beam irradiance. Therefore, for laser beam shaping applications when the output beam phase is important, a second optical element must correct phase distortions introduced by the irradiance redistribution. Frieden computed the shaping of an aspherical refracting surface that would re-collimate the output beam parallel to the optical axis and also to the input beam. Keuzer imposed the constant optical path length condition for all rays passing through the beam shaping optics to control phase variation of the output beam. Unfortunately, optical design and fabrication technologies were generally not adequate until the 1980's to permit realistic design, analysis, fabrication, and testing of laser beam shaping systems.

Today, beam shaping is the process of redistributing the irradiance and phase of a beam of optical radiation. The irradiance distribution defines the beam profile, such as, Gaussian, multimode, annular, rectangular, or circular. The phase of the output beam determines its propagation properties. Contemporary laser beam shaping systems can be grouped into two functional categories: field mappers and beam integrators. A field mapper transforms a known input beam into a desired output beam in a prescribed manner, can be effectively lossless, and works well for single-mode beams. A beam integrator breaks the input beam into a large number of facets by a lens-array, and then, tries to spread the energy within each facet over the output region. The output beam profile is a sum of the diffraction patterns of each lens-array aperture. Beam integrators work well for multimode beams where the input profile may be unknown.

Optical design of beam shaping systems can be achieved using either physical or geometrical optics. There is no single beam shaping method that can be used for all applications. Guidance in choosing a beam shaping technique is discussed in Chapter 1 of Ref. 4. For single-mode Gaussian beams calculating the parameter $\beta$ will help determine the quality of solution available and whether geometrical or physical optics methods should be used

$$
\beta=\frac{2 \sqrt{2 \pi} r_{0} Y_{0}}{f \lambda}
$$

*Other author information: E-mail: dls@uab.edu ; Telephone: 205-934-8068; Fax: 205-934-8042.

Copyright 2002 Society of Photo-Optical Instrumentation Engineers.

This paper will be published in Proc. SPIE 4832 and is made available as an electronic preprint with permission of SPIE. One print or electronic copy may be made for personal use only. Systematic or multiple reproduction, distribution to multiple locations via electronic or other means, duplication of any material in this paper for a fee or for commercial purposes, or modification of the content of the paper are prohibited. 
where $\lambda$ is the wavelength, $\mathrm{r}_{0}$ is the beam radius or waist, $\mathrm{Y}_{0}$ is half-width of the desired output dimension, and $f$ is the focal length of the focusing optic, or the working distance from the optical system to the target plane for systems without a defined focusing optics. For simple output geometries, such as, circles and rectangles, the following rules of thumb have been developed: ${ }^{6}$

- If $\beta<4$, a beam shaping system will not produce acceptable results.

- When $4<\beta<32$, diffraction effects are significant and should be part of design of beam shaping systems.

- When $\beta>32$, geometrical methods should be adequate for design of beam shaping systems.

This paper will summarize the geometrical methods ${ }^{7,8}$ for designing reflective and refractive field mapping systems. Field mapping is basic to all beam shaping, since one seeks to design a set of optical elements that map an input field into a desired output optical field.

For illumination applications, such as in holography, materials processing, and lithography, it is very important for the laser beam to uniformly illuminate the target surface. Both reflective $e^{9,10,11,12}$ and refractive ${ }^{2,13,14,15}$ optical systems have been used to shape laser beam profiles. McDermit and Horton ${ }^{9}$ use conservation of energy within a bundle of rays to design rotationally symmetric reflective optical systems for illuminating a receiver surface in a prescribed manner using a non-uniform input beam profile. Malyak, ${ }^{11}$ Shealy and Chao ${ }^{12}$ have designed a two-mirror laser profile shaping system with rectangular symmetry and no central obscuration which transforms an input Gaussian beam into a uniform irradiance output beam. Kreuzer ${ }^{2}$ has patented a coherent-light optical system using two aspherical surfaces to yield an output beam of desired intensity distribution and wavefront shape. Rhodes and Shealy ${ }^{13}$ derived a set of differential equations using intensity mapping and the constant optical path length condition to calculate the shape of two-aspherical surfaces of a lens system that expands and converts a Gaussian laser beam profile into a collimated, uniform irradiance output beam. Using their method, two-plano-aspherical lenses have been designed, fabricated and used for laser beam shaping in a holographic projection system. ${ }^{16,17,18}$ Hoffnagle and Jefferson ${ }^{15}$ introduced convex aspherical surfaces for ease of fabrication and a continuous roll-off of the output beam profile for more control of the far-field diffraction pattern into their design of a refractive laser beam shaping system.

The theory of geometrical methods for design of a laser beam shaping system is summarized in section 2. A brief overview is presented of the optical design process of incorporating the geometrical optics intensity law for propagation of a bundle of rays and the constant optical path length condition into the ray trace equations, and then, of determining the geometrical contour of several surfaces (or GRIN glasses) so that the beam shaping design conditions are satisfied. Optimization-based techniques, such as, genetic algorithms (GA) have also been shown to be effective methods for design of laser beam shaping systems. ${ }^{19}$ Section 3 summarizes three applications of this theory to the design of laser beam shaping systems - a two plano-aspheric lens system, a two-mirror configuration with no obscuration, and a threeelement GRIN lens system.

\section{THEORY}

In order to optimize the irradiance within an optical system, the optical field must be determined throughout the system. The optical field is a local plane wave solution of Maxwell's equations or the scalar wave equation. ${ }^{20,21}$ For an isotropic, non-conducting, charge-free medium, the optical field may be written as:

$$
u(\mathbf{r})=u_{0}(\mathbf{r}) \exp \left[i k_{0} S(\mathbf{r})\right]
$$

where $\mathrm{k}_{0}=\omega / \mathrm{c}=2 \pi / \lambda_{0}$ is the wave number in free space; $\mathrm{u}_{0}(\mathbf{r})$ and $\mathrm{S}(\mathbf{r})$ are unknown functions of $\mathbf{r}$. Requiring $\mathrm{u}(\mathbf{r})$ from Eq. (2) satisfy the scalar wave equation leads to the following conditions which must be satisfied:

$$
\begin{gathered}
(\nabla S)^{2}=n^{2} \\
2 u_{0} \nabla S \cdot \nabla u_{0}+u_{0}^{2} \nabla^{2} S=\nabla \cdot\left(u_{0}^{2} \nabla S\right)=\nabla \cdot\left(u_{0}^{2} n \mathbf{a}\right)=\nabla \cdot(I \mathbf{a})=0
\end{gathered}
$$

where $\mathrm{n}$ is the index of refraction, and $I$ is the energy density of the field times the speed of propagation within medium. Equation (3) is known as the eikonal equation and is a basic equation of geometrical optics. The surfaces $\mathrm{S}(\mathrm{x}, \mathrm{y}, \mathrm{z})=$ const. are constant phase fronts of the optical field, have a constant optical path length (OPL) from the source or reference 
surface, and are known as the geometrical wavefront. Equation (4) expresses conservation of radiant energy within a bundle of rays and is known as the geometrical optics intensity law for propagation of energy. According to geometrical optics, the phase and amplitude of the optical field are evaluated independently. First, the ray paths are evaluated throughout the optical system with ray tracing. Then, the phase of the optical field is computed from the optical path length of the rays passing through the system. The amplitude (or intensity) of the optical field is computed from the density of rays at any point within the system by monitoring the intensity variations along each ray. ${ }^{22,23,24}$

For beam shaping systems with collimated input and output beams as illustrated in Figure 1, a useful expression for the energy within a bundle of rays ${ }^{25}$ as it passes through the system follows by integrating Eq. (4) over reference planes (or wavefront) normal to the input and output beam and then applying Gauss' theorem

$$
I_{\text {in }} d w=I_{\text {out }} d W .
$$

Equation (5) expresses conservation of energy along a bundle of rays between input element of area and the corresponding output element of area on the wavefront or the reference planes normal to the beam. Equation (5) says that the intensity times the cross-sectional area of the beam is constant along the beam as it propagates through the optical system and is a basic equation used for the optical design of laser beam shaping systems. For some beam shaping configurations, it is necessary to introduce the conservation of energy condition into the optical design by using reference surfaces, such as for detectors, which are curved and/or have an arbitrary orientation with respect to the direction of the beam propagation. In these cases it is necessary to take into account projecting the element of area of a reference surface perpendicular to direction of beam propagation when applying Eq. (5). The total energy of the beam must also be conserved as the beam propagates through the system.

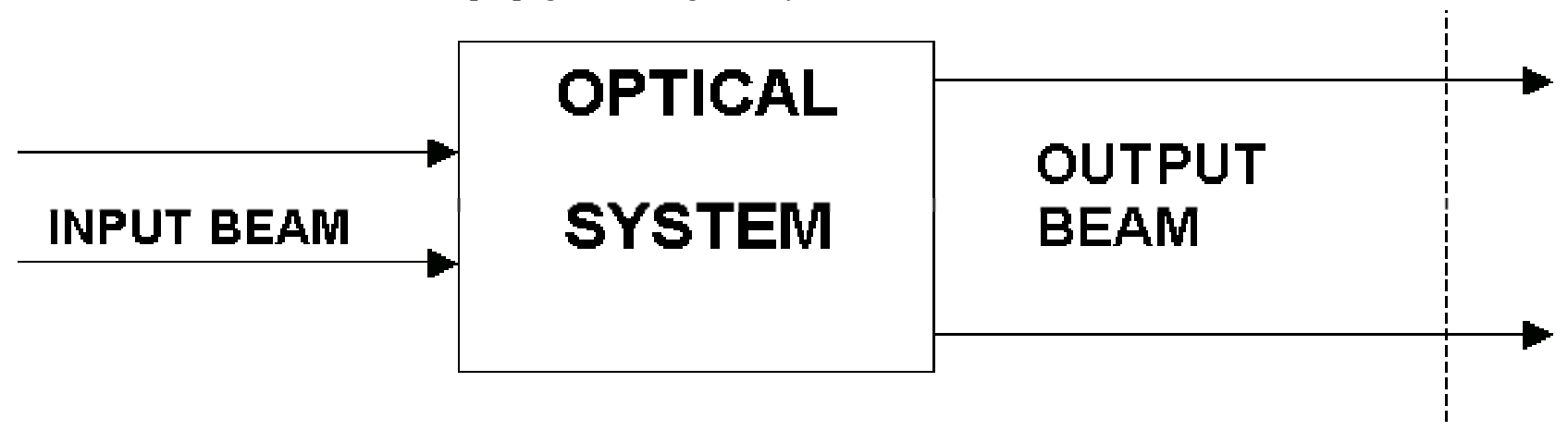

Figure 1. Schematic layout of a laser beam profile shaping system.

When the incident beam is a laser in the fundamental, Gaussian $\mathrm{TEM}_{00}$ mode with central intensity normalized to unity, the input intensity profile is given by

$$
I_{\text {in }}(r)=\exp \left[-2\left(r / r_{0}\right)^{2}\right]
$$

where $\mathrm{r}_{0}$ is the beam radius or waist. This beam leaves the optical system at a radial distance $\mathrm{R}$ from the optical axis with a power density of $I_{\text {out }}(\mathrm{R})$. Integrating Eq. (5) over the input and output planes gives

$$
\int_{0}^{2 \pi} d \theta \int_{0}^{r} I_{\text {in }}(r) r d r=\int_{0}^{2 \pi} d \theta \int_{0}^{R} I_{\text {out }}(R) R d R .
$$

When $I_{\text {out }}(R)$ is constant, the radius of beam in the exit aperture $\mathrm{R}$ can be evaluated by carrying out the integration using Eq. (6) for $\mathrm{I}_{\mathrm{in}}(\mathrm{r})$ to obtain

$$
R=\sqrt{\frac{r_{0}^{2}}{2 I_{\text {out }}}\left[1-\exp \left(-2 r^{2} / r_{0}^{2}\right)\right]} .
$$

The constant $I_{\text {out }}$ (output intensity) is evaluated by integrating Eq. (7) over the working input aperture of radius $r_{\max }$, and the corresponding output aperture, $R_{\max }$, and then, solving for $I_{\text {out }}$ to obtain: 


$$
\mathrm{I}_{\text {out }}=\frac{r_{0}^{2}}{2 R_{\max }^{2}}\left[1-\exp \left(-2 r_{\max }^{2} / r_{0}^{2}\right)\right] .
$$

Equation (8) relates the output beam radius to the input beam and is used during the optical design process to reduce the number of independent variables when solving for the shape of the reflecting or refracting surfaces of the beam shaping system. It is interesting to note that if the output beam profile is a Fermi-Dirac ${ }^{15}$ distribution with a continuous roll-off for more control of the far-field diffraction pattern, it has been shown that application of conservation of energy leads to a nonlinear, analytical relationship between the input and output ray coordinates. ${ }^{26}$

In order to accomplish the overall beam expansion and profile shaping as illustrated in Figure 1, two optical elements are required. These optical elements may be either lenses or mirrors. The first optical element typically expands and shapes the beam profile to satisfy conservation of energy according to Eq. (8), and the second optical element re-collimates the rays so that the constant optical path length condition is satisfied by the system, as illustrated by the two-lens beam shaping system shown in Figure 2. Optical design of a laser beam shaping system seeks to define the optical components adequately so that the system can be analyzed, fabricated, and tested. This generally requires specification of the shape and spacing between the optical surfaces as well as the index of refraction of all the media. For the refracting beam expander system illustrated in Figure 2, the shape of surfaces $\mathrm{s}$ and $\mathrm{S}$ must be determined. The conservation of energy condition and the constant optical path length condition can be solved simultaneously with the ray trace equations for $R(r), z(r), Z(R)$ when $n, d, t_{1}, t_{2}$ are given.

It is interesting to note that several authors ${ }^{7,}{ }^{11,27}$ have shown that the sag of two optical elements of a laser beam shaping system can be expressed as a function of $r$

$$
\begin{gathered}
z(r)=\int f(r) d r+C \\
Z(r)=z(r)+g(r)
\end{gathered}
$$

where $\mathrm{C}$ is a constant, and $f(\mathrm{r})$ and $\mathrm{g}(\mathrm{r})$ are functions defining the optical configurations. Section 3 describes how to design a two-lens, two-mirror, and three-element GRIN laser beam shaping systems.

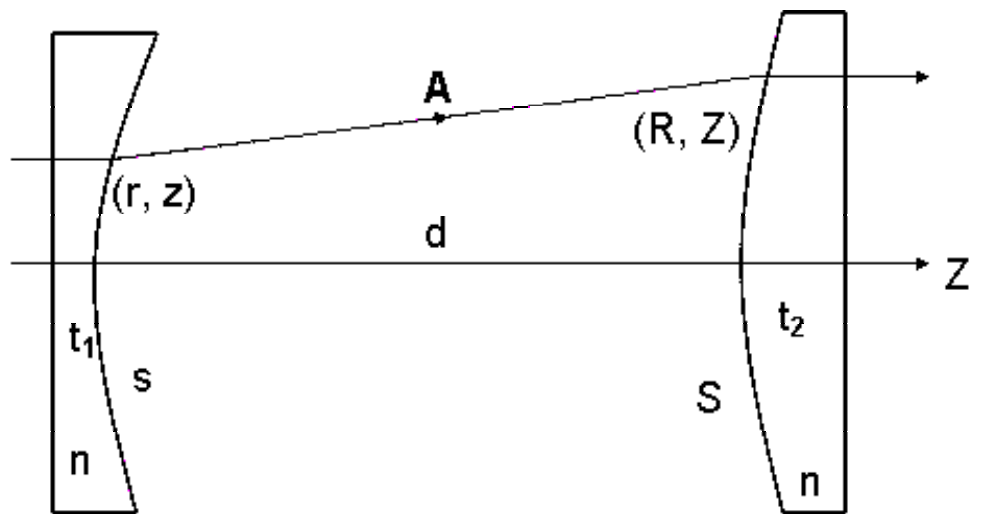

Figure 2. Geometrical configuration of a two-lens laser expander.

\section{Applications}

Using geometrical methods for the optical design of laser beam shaping systems involves incorporating the geometrical optics intensity law for propagation a bundle of rays (conservation of energy) and the constant optical path length condition into the ray trace equations for the optical system, and then, determining the geometrical shapes of several optical surfaces (or GRIN materials) so that the beam shaping design conditions are satisfied. This method of optical design involves solving differential equations for optic shapes or using genetic algorithms to determine configuration parameters of the systems. Three applications - a two-plano-aspheric lens system for shaping a rotationally symmetric Gaussian beam, a two-mirror system with no central obscuration for shaping an elliptical Gaussian input beam, and a 
three-element GRIN system with spherical surfaces for shaping a rotationally symmetric Gaussian beam - are discussed in this section. These applications have been selected to illustrate how the geometrical methods for optical design of laser beam shaping systems are applied to a range of configurations.

\subsection{Two-Lens Beam Shaping System}

The optical design of a two-lens laser beam shaping system with rotational symmetry is summarized in this section. For more details and application of these results, see Refs. 7, 13, 14, 16, 17, 18, and 28. Consider the geometrical configuration of a refracting laser beam profile shaping system shown in Figure 2. The two curved surfaces are used to satisfy the laser beam shaping design conditions. Rays are refracted at surface s according to Snell's law. The ray trace equation of refracted ray $\mathbf{A}$ traveling from the point $(r, z)$ on surface $s$ to the point $(R, Z)$ on surface $S$ is given by

$$
(\mathrm{R}-\mathrm{r})(\mathbf{A})_{z}=(Z-z)(\mathbf{A})_{r}
$$

where ray vector $\mathbf{A}$ is given by

$$
\mathbf{A}=\frac{z^{\prime}\left[n-\sqrt{1+z^{\prime 2}\left(1-n^{2}\right)}\right]}{1+z^{\prime 2}} \mathbf{r}+\frac{\left[n z^{\prime 2}+\sqrt{1+z^{\prime 2}\left(1-n^{2}\right)}\right]}{1+z^{\prime 2}} \mathbf{k} .
$$

Equation (12) can be expressed as a quadratic equation in $z^{\prime}$ and solved to yield

$$
z^{\prime}=\frac{-(R-r)(Z-z) \pm n \sqrt{(Z-z)^{2}+(R-r)^{2}}}{\left[1-n^{2}\right](Z-z)^{2}-n^{2}(R-r)^{2}}
$$

The positive solution for $z^{\prime}$ is used for the lens configuration shown in Figure 2 where the first lens is divergent. For this system, the height of ray $R$ at the second lens with entrance pupil height $r$ is computed from Eq. (8). The term ( $Z$ z) in Eq. (14) is determined by the constant optical path length condition realized by setting the axial optical path length equal to that of a general ray

$$
\left[(R-r)^{2}+(Z-z)^{2}\right]^{1 / 2}=n(Z-z)-d(n-1),
$$

which is a quadratic equation for the term $(\mathrm{Z}-\mathrm{z})$ as a function of the entrance pupil aperture radius $\mathrm{r}$. After squaring Eq. (15) and collecting terms, the solution of the resulting quadratic equation is

$$
(Z-z)=\frac{n(n-1) d+\left[(n-1)^{2} d^{2}+\left(n^{2}-1\right)(R-r)^{2}\right]^{1 / 2}}{n^{2}-1}
$$

where the positive sign of the radical has been used so that the solution reduces to the appropriate value of $(Z-z)=d$ when $r=R=0$. It is interesting to note that Eq. (14) permits $z^{\prime}$ to be expressed as a function of $r$, thus, enabling $z(r)$ to be evaluated by integration, as illustrated in Eq. (10). Reference 28 presents results for design, fabrication, and testing of a two-lens laser beam shaping system similar to configuration shown in Figure 2.

\subsection{Two-Mirror Beam Shaping System}

The optical design of a two-mirror laser beam shaping system with rectangular symmetry will be summarized in this section. For more details and applications of these results, see Refs. 7, 11, and 12. Consider the two-mirror laser beam shaping system illustrated in Figure 3. The input and output beams are collimated and parallel to the optical axis. Assume the input beam has an elliptical cross-section, and its irradiance is given by

$$
I_{\text {in }}(x, y)=\exp \left[-2\left(\frac{x}{x_{0}}\right)^{2}\right] \exp \left[-2\left(\frac{y}{y_{0}}\right)^{2}\right]
$$

where $\left(\mathrm{x}_{0}, \mathrm{y}_{0}\right)$ are the beam waist in the $\mathrm{x}, \mathrm{y}$ directions, respectively, and the central intensity is normalized to unity. The output beam irradiance is uniform and is given by

$$
I_{\text {out }}=A_{X_{0}} A_{Y_{0}}=\text { const. }
$$




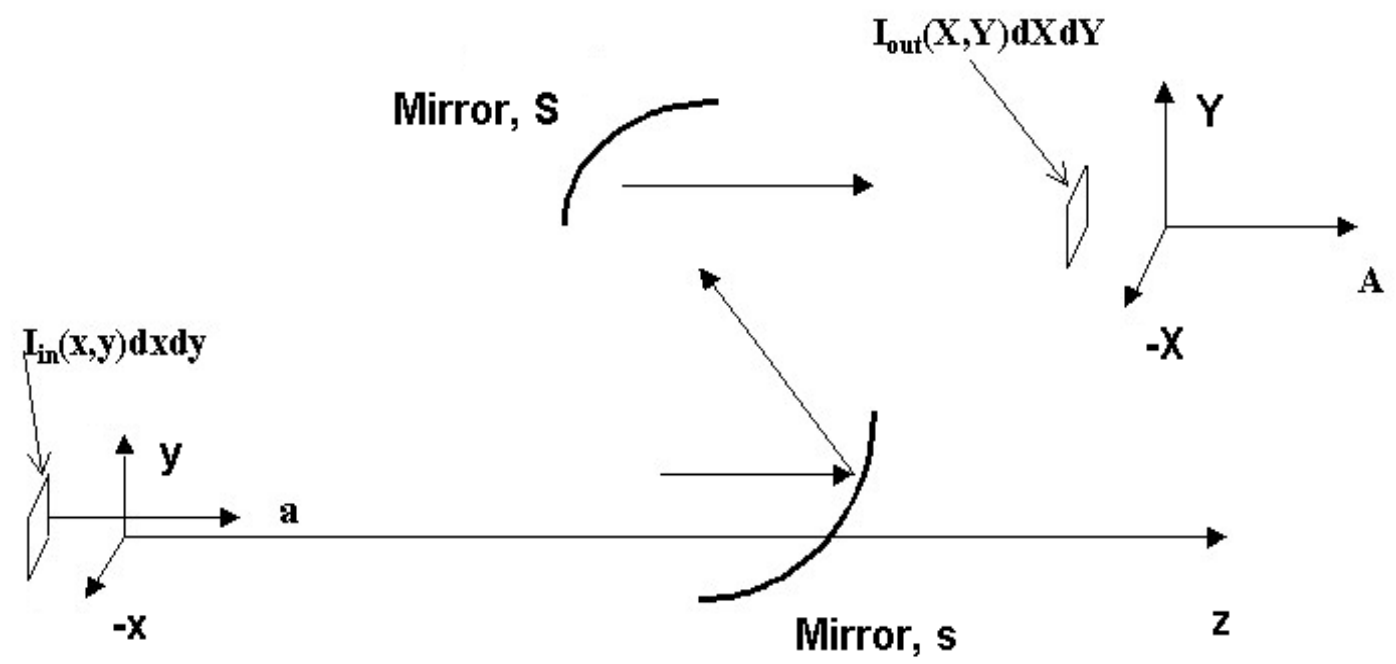

Figure 3. Geometrical configuration of a two-mirror laser beam shaping system with rectangular symmetry. (From Ref. 12)

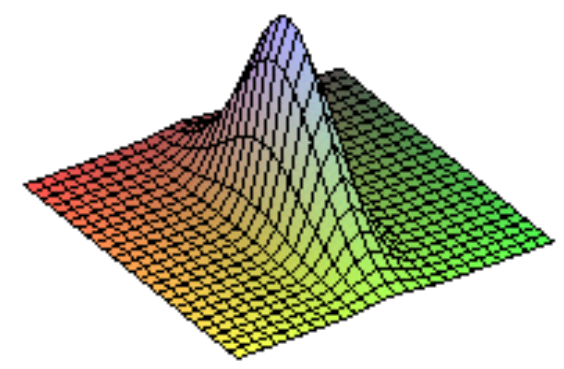

Figure 4. Elliptical Gaussian input beam profile.

Consider the specific input beam as illustrated in Figure 4 with waist and output beam size given by

$$
x_{0}=\frac{p_{0}}{2} ; \quad x_{\max }=p_{0} ; \quad y_{0}=\frac{3 p_{0}}{2} ; \quad y_{\max }=3 p_{0} ; \quad X_{\max }=2 p_{0}=Y_{\max },
$$

where the scaling parameter $\mathrm{p}_{0}$ for linear (transverse) dimensions has been introduced for convenience. The relationship between the element of areas on the input and output planes can be written in the following form

$$
I_{\text {in }}(x, y) d x d y=I_{\text {out }} d X d Y \text {. }
$$

The total energy must also be conserved which follows integrating Eq. (20) over the full aperture on the input and output planes

$$
E_{\text {in }}=\left\{\int_{-x_{\max }}^{x_{\max }} \exp \left[-2\left(\frac{x}{x_{0}}\right)^{2}\right] d x\right\}\left\{\int_{-y_{\max }}^{y_{\max }} \exp \left[-2\left(\frac{y}{y_{0}}\right)^{2}\right] d y\right\}=\left\{\int_{-X_{\max }}^{X_{\max }} A_{X_{0}} d X\right\}\left\{\int_{-Y_{\max }}^{Y_{\max }} A_{Y_{0}} d Y\right\} .
$$

or explicitly after evaluating the integrals

$$
\mathrm{E}_{\mathrm{in}}=\left\{\frac{p_{0} \sqrt{2 \pi}}{4} \operatorname{erf}(2 \sqrt{2})\right\}\left\{\frac{3 p_{0} \sqrt{2 \pi}}{4} \operatorname{erf}(2 \sqrt{2})\right\}=16 I_{\text {out }} p_{0}^{2} .
$$


Solving Eq. (22) for $I_{\text {out }}$ gives

$$
I_{\text {out }}=\frac{3 \pi}{128}[\operatorname{erf}(2 \sqrt{2})]^{2}=0.07362175017 \text {. }
$$

From Eq. (21) expressions for the output irradiance along the $\mathrm{x}, \mathrm{y}$ directions are given by

$$
\begin{gathered}
A_{X_{0}}=\frac{1}{4 p_{0}} \int_{p_{0}}^{p_{0}} \exp \left[-2\left(\frac{2 x}{p_{0}}\right)^{2}\right] d x=\frac{\sqrt{2 \pi}}{16} \operatorname{erf}(2 \sqrt{2}), \\
A_{Y_{0}}=\frac{1}{4 p_{0}} \int_{-3 p_{0}}^{3 p_{0}} \exp \left[-2\left(\frac{2 y}{3 p_{0}}\right)^{2}\right] d x=\frac{3 \sqrt{2 \pi}}{16} \operatorname{erf}(2 \sqrt{2}) .
\end{gathered}
$$

Since there is a non-uniform shaping of the laser beam profile in orthogonal directions, assume there is an independent and non-uniform magnification of the $\mathrm{x}$ and $\mathrm{y}$ ray coordinates between the input and output planes:

$$
\begin{aligned}
& X=m_{x}(x) x, \\
& Y=m_{y}(y) y .
\end{aligned}
$$

The rectangular magnifications $\mathrm{m}_{\mathrm{x}}(\mathrm{x})$ and $\mathrm{m}_{\mathrm{y}}(\mathrm{y})$ can be determined by imposing the incremental expression of conservation of energy, Eq. (20), for the intensity functions, Eqs. (17) and (18), and then separating variables to obtain

$$
\frac{\exp \left[-2\left(\frac{x}{x_{0}}\right)^{2}\right]}{A_{X_{0}}\left(\frac{\partial\left[m_{x}(x) x\right]}{\partial x}\right)}=\frac{A_{Y_{0}}}{\exp \left[-2\left(\frac{y}{y_{0}}\right)^{2}\right]} \frac{\partial\left[m_{y}(y) y\right]}{\partial y}=\frac{1}{C_{1}}, \text { const. }
$$

Setting the right- and left-hand side Eq. (28) equal to the constant, and integrating leads to expressions for the magnifications of the ray coordinates on the output plane. Then, using Eqs. (26) and (27), the following expressions for the ray coordinates on the output plane follow

$$
\begin{aligned}
& X=\frac{1}{A_{X_{0}}} \int_{0}^{x} \exp \left[-2\left(\frac{2 u}{p_{0}}\right)^{2}\right] d u=2 p_{0} \frac{\operatorname{erf}\left(2 \sqrt{2}\left(\frac{x}{p_{0}}\right)\right)}{\operatorname{erf}(2 \sqrt{2})} \\
& Y=\frac{1}{A_{Y_{0}}} \int_{0}^{y} \exp \left[-2\left(\frac{2 v}{3 p_{0}}\right)^{2}\right] d v=2 p_{0} \frac{\operatorname{erf}\left(\frac{2 \sqrt{2}}{3}\left(\frac{y}{p_{0}}\right)\right)}{\operatorname{erf}(2 \sqrt{2})}
\end{aligned}
$$

where $\mathrm{C}_{2}$ and $\mathrm{C}_{3}$ are constants determined by the boundary conditions. Equations (29) and (30) can be used to evaluate numerical tables relating $(\mathrm{x}, \mathrm{y})$ to $(\mathrm{X}, \mathrm{Y})$, which can then be used to invert numerically the magnification equations (26) and (27) so that one can express (x, y) in terms of (X, Y) when evaluating $Z(X, Y)$ in Eq. (50) below.

Now, the ray trace equations connecting two mirror surfaces $s[x, y, z(x, y)]$ and $S[X, Y, Z(X, Y)]$ can be written in the following form: 


$$
\begin{gathered}
\frac{\left(X_{0}+X-x\right)}{\left(Y_{0}+Y-y\right)}=\frac{\left(\mathbf{A}_{1 \rightarrow 2}\right)_{x}}{\left(\mathbf{A}_{1 \rightarrow 2}\right)_{y}}=\frac{z_{x}}{z_{y}} \\
\frac{\left(X_{0}+X-x\right)}{\left(Z_{0}+Z-z\right)}=\frac{\left(\mathbf{A}_{1 \rightarrow 2}\right)_{x}}{\left(\mathbf{A}_{1 \rightarrow 2}\right)_{z}}=\frac{-2 z_{x}}{\left(1-z_{x}^{2}-z_{y}^{2}\right)}
\end{gathered}
$$

where $\mathbf{A}_{1 \rightarrow 2}$ is a unit vector along the ray path connecting the points on the two mirror surfaces $\mathrm{s}[\mathrm{x}, \mathrm{y}, \mathrm{z}(\mathrm{x}, \mathrm{y})]$ and $\mathrm{S}[\mathrm{X}$, $\mathrm{Y}, \mathrm{Z}(\mathrm{X}, \mathrm{Y})]$. The point $\left(\mathrm{X}_{0}, \mathrm{Y}_{0}, \mathrm{Z}_{0}\right)$ locates the origin of the local coordinate system expressed in terms of the variables $(\mathrm{X}, \mathrm{Y}, \mathrm{Z})$, of the second mirror relative to the local coordinate system of the first mirror, $(\mathrm{x}, \mathrm{y}, \mathrm{z})$. The displacement vector,

$$
\mathbf{R}_{0}=X_{0} \mathbf{i}+Y_{0} \mathbf{j}+Z_{0} \mathbf{k},
$$

specifies the location of the vertex of the second mirror with respect to the vertex of the first mirror. The equation of each mirror surface is normally expressed in terms of a local coordinate system whose origin is at the intersection of each mirror with respect to the optical axis and can be written in the following form:

$$
\begin{aligned}
& z=z(x, y), \\
& Z=Z(X, Y) .
\end{aligned}
$$

It will be convenient to express subsequent equations in a more compact form in terms of the position vectors $\mathbf{r}, \mathbf{R}$ of the point of reflection of a ray from the first and second mirror surfaces:

$$
\begin{aligned}
& \mathbf{r}(x, y)=x \mathbf{i}+y \mathbf{j}+z(x, y) \mathbf{k} \\
& \mathbf{R}(X, Y)=X \mathbf{i}+Y \mathbf{j}+Z(X, Y) \mathbf{k}
\end{aligned}
$$

Applying the law of reflection at the first mirror surface for a collimated input beam parallel to the optical axis leads to the following expression for the unit vector $\mathbf{A}_{1 \rightarrow 2}$ :

$$
\mathbf{A}_{1 \rightarrow \mathbf{2}}=\mathbf{a}-2 \hat{\mathbf{n}}(\mathbf{a} \cdot \hat{\mathbf{n}})=\frac{-2 z_{x} \hat{\mathbf{i}}-2 z_{y} \hat{\mathbf{j}}+\left(1-z_{x}^{2}-z_{y}^{2}\right) \hat{\mathbf{k}}}{\left[1+z_{x}^{2}+z_{y}^{2}\right]}
$$

where

$$
\mathbf{a}=\hat{\mathbf{k}} ; \quad \hat{\mathbf{n}}=\frac{-z_{x} \hat{\mathbf{i}}-z_{y} \hat{\mathbf{j}}+\hat{\mathbf{k}}}{\sqrt{1+z_{x}^{2}+z_{y}^{2}}} ; \quad z_{x}=\frac{\partial z(x, y)}{\partial x} \text { and } z_{y}=\frac{\partial z(x, y)}{\partial y}
$$

Eliminating $z_{y}$ between the ray trace equations (31) and (32) leads to a quadratic equation in $z_{x}$ given below

$$
z_{x}^{2}\left[\left(X_{0}+X-x\right)^{2}\right]-2 z_{x}\left(X_{0}+X-x\right)\left(Z_{0}+Z-z\right)-\left(X_{0}+X-x\right)^{2}=0
$$

whose solution is given by the quadratic formula

$$
z_{x}=\left(X_{0}+X-x\right)\left\{\frac{\left[Z_{0}+Z(X, Y)-z(x, y)\right] \pm\left|\mathbf{R}_{0}+\mathbf{R}-\mathbf{r}\right|}{\left(X_{0}+X-x\right)^{2}+\left(Y_{0}+Y-y\right)^{2}}\right\} .
$$

where

$$
\left|\mathbf{R}_{0}+\mathbf{R}-\mathbf{r}\right|=\sqrt{\left(X_{0}+X-x\right)^{2}+\left(Y_{0}+Y-y\right)^{2}+\left(Z_{0}+Z(X, Y)-z(x, y)\right)^{2}} .
$$


Equation (39) is a partial differential equation for the unknown mirror sag functions $\mathrm{z}(\mathrm{x}, \mathrm{y})$ and $\mathrm{Z}(\mathrm{X}, \mathrm{Y})$. An expression for $z_{y}$ follows from the ray trace equations (31)

$$
z_{y}=z_{x} \frac{\left(Y_{0}+Y-y\right)}{\left(X_{0}+X-x\right)} .
$$

Up to this point, conservation of energy within a bundle of rays has been used to express the output ray coordinates (X, $\mathrm{Y}$ ) as a function of the input ray coordinates (x, y) and beam intensity profiles, according to Eqs. (29) and (30). The ray trace equations (31) and (32) have lead to partial differential equations for the sag function $\mathrm{z}(\mathrm{x}, \mathrm{y})$ of the first mirror surface and the unknown equation of the second mirror surface $\mathrm{Z}(\mathrm{X}, \mathrm{Y})$. To insure that the input and output wavefronts have the same shapes, e.g., plane wavefronts, the phase of all rays passing through the beam shaping system must be constant. Thus, the constant optical path length condition must also be used as part of the optical design of this twomirror laser beam shaping system.

The constant optical path length (OPL) condition provides another independent condition, which can be used to solve for the sag functions $\mathrm{z}(\mathrm{x}, \mathrm{y})$ and $\mathrm{Z}(\mathrm{X}, \mathrm{Y})$ of the two mirror surfaces. The OPL of an axial ray and general ray are given by

$$
\begin{gathered}
(O P L)_{\text {Axial Ray }}=\sqrt{\mathrm{X}_{0}^{2}+\mathrm{Y}_{0}^{2}+\mathrm{Z}_{0}^{2}} \equiv R_{0}, \\
(O L P)_{\text {General Ray }}=\left|\mathbf{R}_{0}+\mathbf{R}-\mathbf{r}\right|+Z(X, Y)-z(x, y)
\end{gathered}
$$

where the axial ray is incident upon the first mirror at its vertex on the optical axis and origin of the local coordinate system $(\mathrm{x}, \mathrm{y}, \mathrm{z})$ and is reflected to the vertex and the origin of the local coordinate system $(\mathrm{X}, \mathrm{Y}, \mathrm{Z})$ on the second mirror. The OPL of the non-axial ray is equal to the distance between the points of reflection on each mirror, $\left|\mathbf{R}_{0}+\mathbf{R}-\mathbf{r}\right|$, plus the difference between the sag function of the point of reflection on each mirror, $\mathrm{Z}(\mathrm{X}, \mathrm{Y})-\mathrm{z}(\mathrm{x}, \mathrm{y})$. Equating the right-hand-side of Eqs. (42) and (43) leads to the following:

$$
R_{0}-(Z(X, Y)-z(x, y))=\left|\mathbf{R}_{0}+\mathbf{R}-\mathbf{r}\right| .
$$

The constant optical path length equation (44) can be used to express $z_{x}$ from Eq. (39) as a function of $(x, X)$ by squaring Eq. (44) to display

$$
\begin{aligned}
\left(X_{0} X-x\right)^{2}+ & \left(Y_{0}+Y-y\right)^{2}=R_{0}^{2}-Z_{0}^{2}-2\left(Z_{0}+R_{0}\right)(Z-z) \\
& =\left(R_{0}+Z_{0}\right)\left[R_{0}-Z_{0}-2(Z(X, Y)-z(x, y))\right]
\end{aligned}
$$

Using the negative sign in Eq. (39) as the physically meaningful solution, and combining Eqs. (31), (32), (39), (44), and (45) gives the following expressions

$$
z_{x}=\frac{\left(x-X-X_{0}\right)}{\left(R_{0}+Z_{0}\right)} ; \quad z_{y}=\frac{\left(y-Y-Y_{0}\right)}{\left(R_{0}+Z_{0}\right)} .
$$

Since $\mathrm{X}=\mathrm{X}(\mathrm{x})$ and $\mathrm{Y}=\mathrm{Y}(\mathrm{y})$ from Eqs. (31) and (32), then it follows that the sag of the first mirror can be written as

$$
\begin{aligned}
z(x, y) & =\int z_{x}(x, y) d x+\int z_{y}(x, y) d y \\
& =\frac{1}{\left(R_{0}+Z_{0}\right)}\left\{\int_{0}^{x}[u-X(u)] d u+\int_{0}^{y}[v-Y(v)] d v+C_{4}\right\},
\end{aligned}
$$

where $\mathrm{C}_{4}$ is a constant of integration. After explicit integration of Eq. (47), the sag of the first mirror is given by 


$$
z(x, y)\left(R_{0}+Z_{0}\right)=\frac{1}{2}\left(x^{2}+y^{2}\right)+\frac{p_{0}^{2}}{\sqrt{2 \pi} \operatorname{erf}(2 \sqrt{2})} \omega(x, y)+C_{4}
$$

where

$$
\omega(x, y)=\left\{4-\exp \left[-2\left(\frac{2 x}{p_{0}}\right)^{2}\right]-3 \exp \left[-2\left(\frac{2 y}{3 p_{0}}\right)^{2}\right]-\frac{2 \sqrt{2 \pi}}{p_{0}}\left[x \operatorname{erf}\left(\frac{2 \sqrt{2} x}{p_{0}}\right)+y \operatorname{erf}\left(\frac{2 \sqrt{2} y}{3 p_{0}}\right)\right]\right\} .
$$

The sag of the second mirror surface can now be evaluated from Eq. (45) to give

$$
Z(X, Y)=\left[z(x, y)+\frac{-\left(x-X(x)-X_{0}\right)^{2}-\left(y-Y(y)+Y_{0}\right)^{2}+R_{0}^{2}-Z_{0}^{2}}{2\left(Z_{0}+R_{0}\right)}\right]_{\substack{x=m_{x}^{-1}(X) X \\ y=m_{y}^{-1}(Y) Y}}
$$

where the $\mathrm{x}$ and $\mathrm{y}$ terms are eliminated from Eq. (50) by inverting numerically the ray output coordinates equations (29) and (30). The process of evaluating the sag of each mirror surface is discussed in detail in Ref. 12.

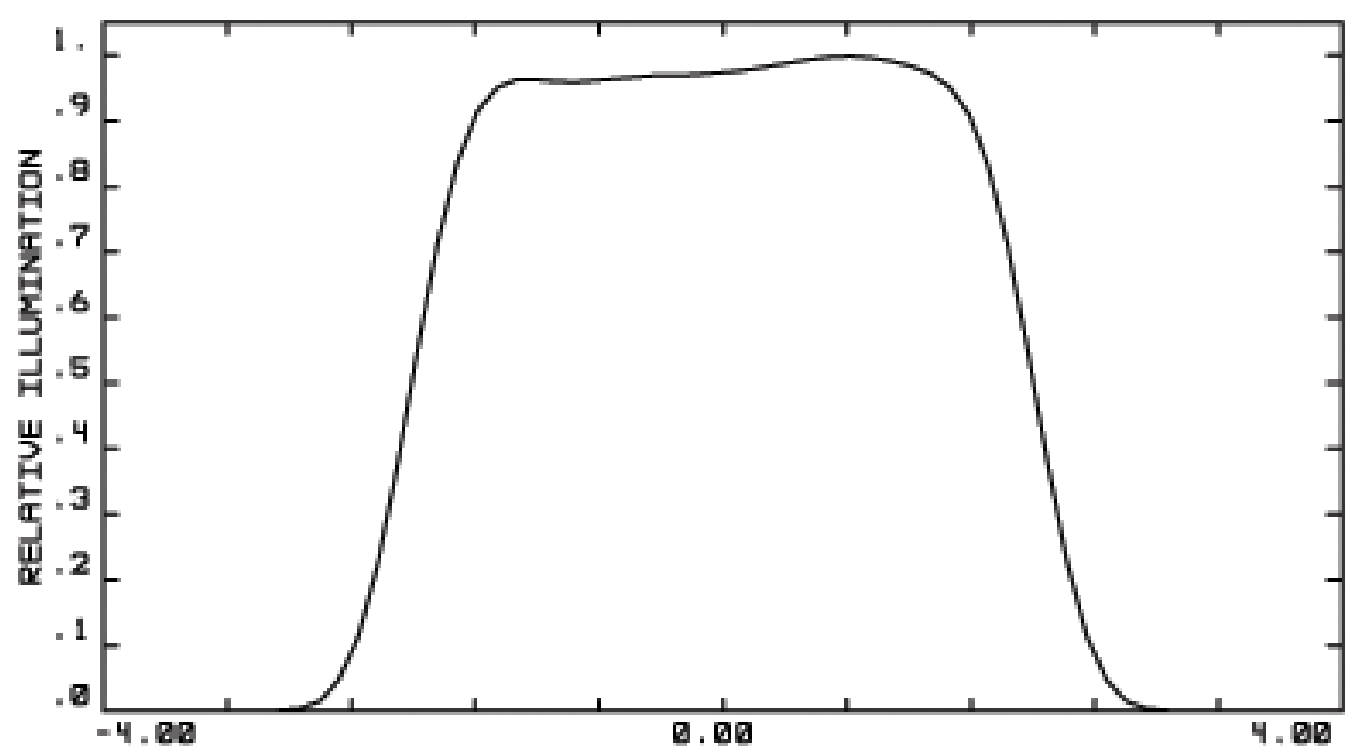

Figure 5. Relative Illumination of the output beam along the x-y axes. (From Ref. 12)

Using these results, the performance of a two-mirror laser beam shaping system, which transforms an input beam with elliptical cross section of 3:1 ratio of beam waist in perpendicular directions, has been analyzed. ${ }^{12}$ The relative output illumination is shown in Figure 5. The aspherical deviation of the mirror surfaces from best-fit sphere has been shown to be $120 \mu \mathrm{m}$ for a $6 \mathrm{~mm}$ diameter mirror. The optical analysis software ZEMAX ${ }^{29}$ has been used for performance modeling and tolerance analysis of this system. These results show that the first mirror surface has a strong aspherical component along the direction of smaller input beam waist and that the output beam profile remains fairly uniform when the mirror decentation is less than $2.5 \%$ of the maximum mirror surface dimension and tiltation is less than 2.5 degrees about the coordinate axis.

\section{3. $\quad$ Three GRIN Element Laser Beam Shaping System}

This application was motivated by the desire to design a spherical-surface GRIN lens system, where catalog GRIN glass types are used and the number of lens element required is an optimization variable. Solution to this problem will 
represent a way to construct a laser beam shaping system without use of aspherical optics or esoteric GRIN profiles. ${ }^{30}$ The optical design for this system involves using genetic algorithms (GA) to maximize a laser beam shaping merit function, Eq. (51), within a 26-dimensional parameter space. ${ }^{31}$ To solve this optical design problem, the GA not only must optimize surface shapes of the GRIN elements and their spacing, but also must determine the actual number of GRIN elements in the solution, up to a certain limit (four, in this case), and the type of GRIN material for each element must be selected from a vendor GRIN glass catalog. This type of problem distinguishes the GA method from deterministic methods (i.e., those that rely on derivatives and a smooth, continuous merit function) since the merit function required for this problem depends on a complicated mix of discrete and continuous parameters. For more details and applications of these results, see Refs. 19, 31, and 32.

The optical design of this system solves for the attributes (radii, thickness, and spacing) of the lens elements, the GRIN glass type from a catalog, and the number of elements needed for a system of this configuration to satisfy the beam shaping design conditions - conservation of energy within beam and constant optical path length condition. Twenty-six parameters of the lens system are determined by the GA optimization. The merit function M includes terms that favor a specific beam diameter, a uniform irradiance output beam profile, and a collimated output beam. The following merit function satisfies these design objectives and is maximized during design:

$$
M=\frac{M_{\text {Diameter }} M_{\text {Collimation }}}{M_{\text {Uniformity }}}=\frac{\exp \left[-s\left(R_{\text {Target }}-R_{\mathrm{N}}\right)^{2}\right] \exp \left[-\left(1-\prod_{i=1}^{N} \cos ^{Q}\left(\gamma_{i}\right)\right)^{2}\right]}{\sqrt{\frac{1}{N} \sum_{i=1}^{N}\left\{I_{\text {out }}\left(R_{i}\right)-\left[\frac{1}{N} \sum_{k=1}^{N} I_{\text {out }}\left(R_{k}\right)\right]\right\}}}
$$

where $R_{\text {Target }}$ is the desired radius of the output beam, $R_{N}$ is the radial height of the marginal ray on the output surface, $\gamma_{i}$ is the angle the $\mathrm{i}^{\text {th }}$ ray makes with the optical axis, $\mathrm{s}$ and $\mathrm{Q}$ are convergence constants used to adjust importance of different components of merit function during optimization, and $\mathrm{I}_{\text {out }}\left(\mathrm{R}_{\mathrm{k}}\right)$ is the irradiance of ray in output beam. (See Ref. 32 for a detailed discussion of this GA optimization process and construction of a suitable merit function to use when designing laser beam shaping systems.) The exponential function is used in the merit function, since it peaks strongly as parameters approach their design targets. Also, as the output beam profile becomes more uniform, the denominator of Eq. (51) approaches zero, and $M$ increases substantially. In summary, the merit function rewards those systems, which tend to increase the value of $M$ and penalize systems with smaller values of $M$ as the GA optimization searches throughout both the discrete and continuous parameter space. After 12,367 generations (iterations), the GA converges to a three-element GRIN lens system with all spherical surfaces which is illustrated in Figure 6.

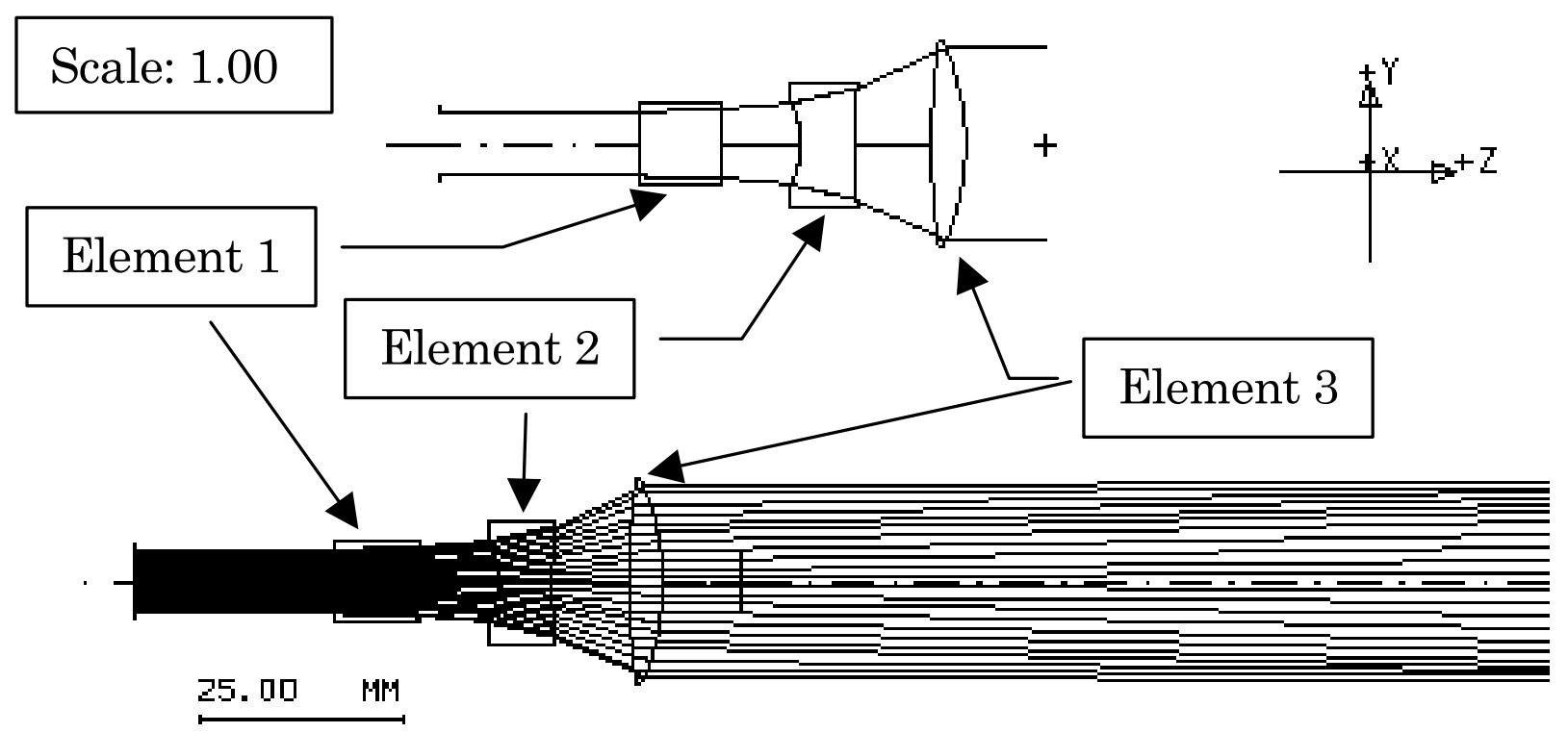

Figure 6. Raytrace for the free-form GA-designed GRIN shaper system. (From Ref.31) 
The code was executed on a Sun Ultra 1170 with $64 \mathrm{M}$ of RAM. Using this setup, it took an average of 7.80 seconds for the GA to completely evaluate the merit function for the 10 test systems (individuals) in a generation. The code was allowed to run until it reached generation number 12,367, resulting in an effective total run-time of 26.8 hours. The merit function, which is measured in arbitrary units, peaked at a value of $M=101.21$. The fact that this value represents a convergent solution to the problem can be seen in Figure 7, where it is clear that $\mathrm{M}_{\text {best }}$ is asymptotically approaching a value of about 102. The system with a merit function value of $M=101.21$ is represented in Figure 6 . The system has three elements, all with spherical surfaces. This solution shapes the Gaussian input beam profile into a uniform irradiance profile on the output surface and conserves energy. To check conservation of energy for this GA optimization process, integrate the output irradiance profile over the output surface and compare this result to the integral of the input irradiance over the input surface. For the configuration shown in Figure 6, the mean value for the output irradiance is $4.55 \times 10^{-2}$ rays per $\mathrm{mm}^{2}$ with a standard deviation of $1.7 \times 10^{-3}$ rays per $\mathrm{mm}^{2}$ or $3.7 \%$. The integral of the output irradiance over the output surface yields 21.9 units, while integrating the input irradiance profile over the input surface yields 21.7 units or within computational errors of the output irradiance. The input and output profiles are shown in Figure 8. The parameters for this system are given in Table 1 and Table 2.

To review, the GA-based laser beam shaping method produced a three-element laser beam shaping system with spherical surfaces for optics, and uses GRIN elements from the catalog of an established GRIN materials manufacturer. The GA solved a problem that would be difficult to solve using analytical methods or conventional optimization techniques, since the merit function contains discrete parameters (for example, picking the GRIN glass type from a predefined set of GRIN elements). Furthermore, the GA was presented with a unique problem that has not been solved before and was allowed a certain degree of creativity in producing a solution.

\section{SUMMARY AND CONCLUSIONS}

The geometrical methods for design of laser beam shaping systems have been reviewed. This geometrical optics-based theory for designing laser beam shaping is based on conservation of radiant energy within a bundle of rays, the ray trace equations, and the constant optical path length condition for cases when contour of the incident wavefront is maintained as the beam passes through the system. This theory has been used to compute the sag of the optical surfaces for twoplano-aspheric lens system and a two-mirror configuration and to setup a laser beam shaping merit function for a genetic algorithm optimization of a three-element GRIN system with spherical surfaces.

In the first example, numerical techniques were used to solve the differential equations for the sag of the shaping elements in a two-lens laser beam shaping system, which has been fabricated and tested. In the second example, analytical and numerical techniques were used to determine and analyze the performance of a two-mirror laser beam shaping system with no central obscuration, which can transform an elliptical Gaussian input beam into a rectangularsymmetric output beam with uniform irradiance. The optical analysis software ZEMAX has been used for performance modeling and tolerance analysis for this system. These results show that the first mirror surface has a strong aspherical component along the direction of smaller input beam waist and that the output beam profile remains fairly uniform when the mirror decentation is less than $2.5 \%$ of the maximum mirror surface dimension and tiltation is less than 2.5 degrees about the coordinate axis.

In the last example, genetic algorithms (GA) were used to design a spherical-surface GRIN lens laser beam shaping system, where catalog GRIN glass types are used and the number of lens element required is an optimization variable. The optical design for this system involves maximizing a merit function based on output beam irradiance, size, and direction of propagation within a 26-dimensional parameter space. To solve this optical design problem, the GA optimized surface shapes of the GRIN elements and their spacing, determined the actual number of GRIN elements in the solution, up to a certain limit (four, in this case), and the type of GRIN material for each element was selected from a GRIN glass catalog. These applications show that the geometrical methods for design of range of laser beam shaping systems are effective for these types of configurations. 


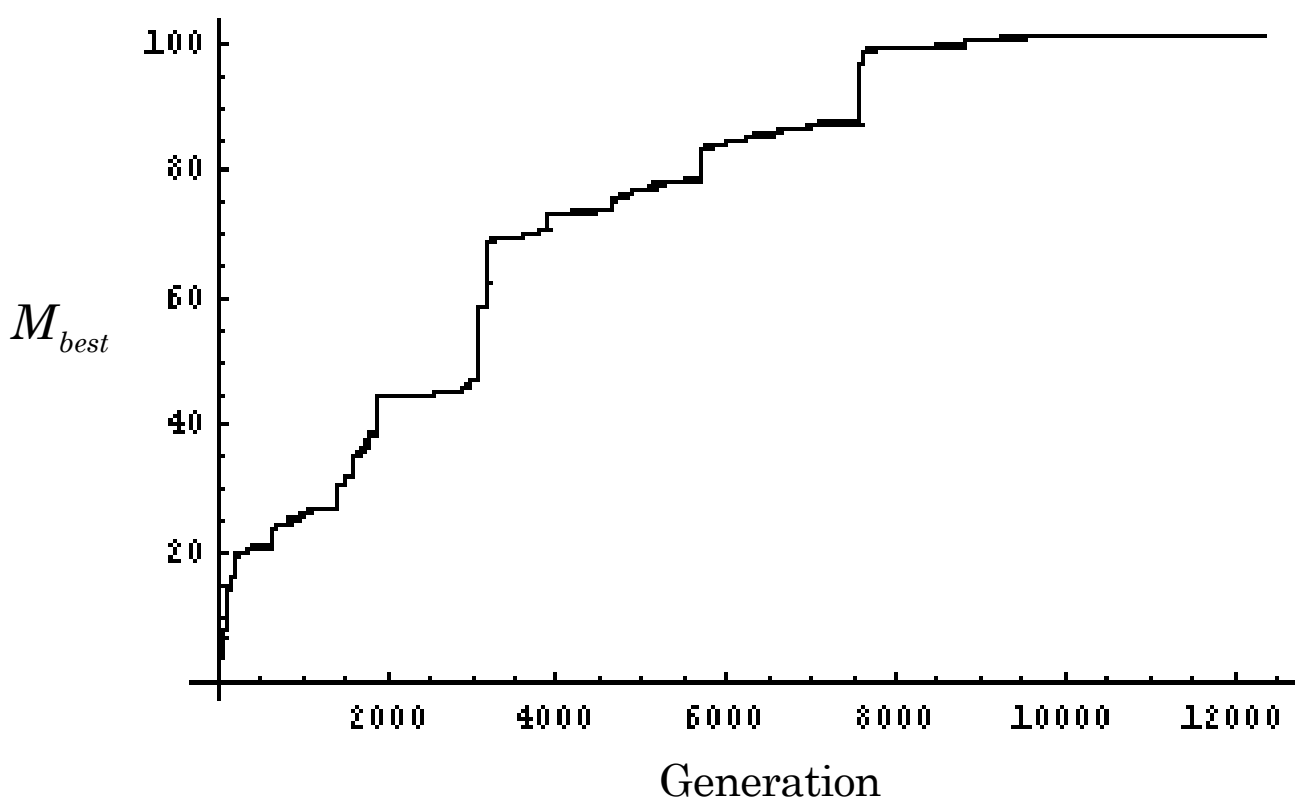

Figure 7. A plot showing the best individual in a generation as a function of generation. (From Ref. 31)

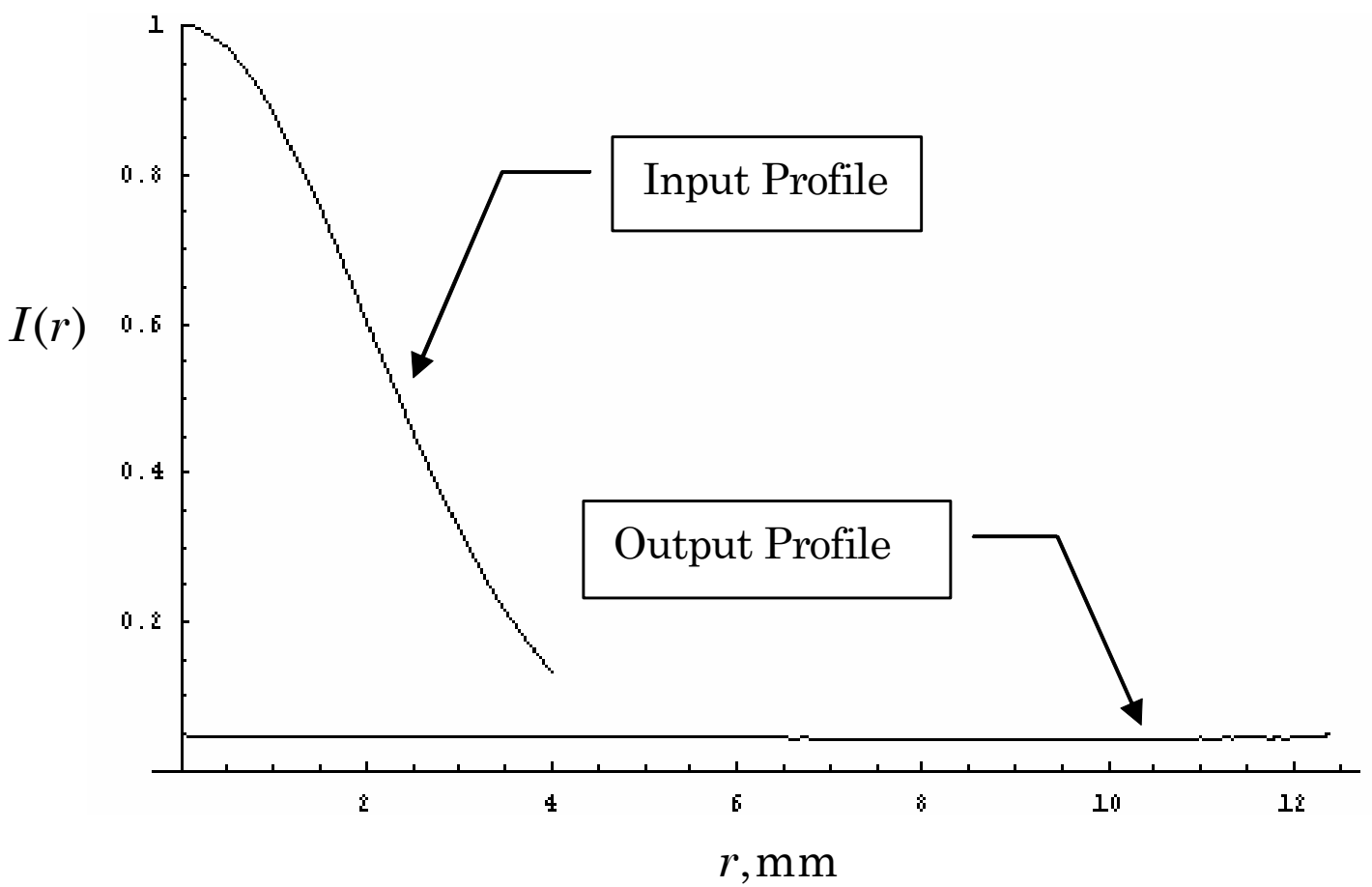

Figure 8. Input and output irradiance profiles for the three-element GA-designed GRIN laser beam shaping system. (From Ref. 31) 
Table 1. $\quad$ Free-form GA-Designed GRIN Shaper Parameters (From Ref. 31)

\begin{tabular}{ll}
\hline Parameter & Value \\
\hline Wavelength & $589.00 \mathrm{~nm}$ \\
Radius of the input beam (entrance pupil diameter) & $4.00 \mathrm{~mm}$ \\
Radius of the output aperture & $12.4 \mathrm{~mm}$ \\
Index of ambient medium (air) & 1.0 \\
Gaussian constant $2 / r_{0}^{2}$ & $0.035 \mathrm{~mm}^{-2}$ \\
Number of elements & 3 \\
Distance from Surface 10 to output plane (parameter 26 in Table 8, Ref. 31) & $100 \mathrm{~mm}$ \\
Object distance & Infinity \\
\hline
\end{tabular}

Table 2. $\quad$ Free-form GA-Designed GRIN Shaper Lens Parameters (From Ref. 31)

\begin{tabular}{lllllll}
\hline \multirow{2}{*}{ Parameter } & \multicolumn{2}{l}{ First element } & \multicolumn{2}{l}{ Second element } & \multicolumn{2}{l}{ Third element } \\
\cline { 2 - 7 } & $\begin{array}{l}\text { Left } \\
\text { surface }\end{array}$ & $\begin{array}{l}\text { Right } \\
\text { surface }\end{array}$ & Left surface & $\begin{array}{l}\text { Right } \\
\text { surface }\end{array}$ & Left surface & $\begin{array}{l}\text { Right } \\
\text { surface }\end{array}$ \\
\hline Thickness, mm & 9.99 & 10.0 & 6.77 & 9.48 & 4.25 & 9.71 \\
Vertex radius, mm & -61.6 & 80.4 & -12.5 & 100 & 87.5 & -30.3 \\
Surface type & spherical & spherical & spherical & spherical & spherical & spherical \\
Glass Type (UDG C1) & 3 & & 2 & & 1 & \\
GRIN Direction & negative & & positive & & negative & \\
\hline
\end{tabular}

\section{REFERENCES}

1 B. R. Frieden, "Lossless conversion of a plane laser wave to a plane wave of uniform irradiance," Appl. Opt. 4.11, pp. 1400-1403, 1965.

2 J. L. Kreuzer, "Coherent light optical system yielding an output beam of desired intensity distribution at a desired equiphase surface," U. S. Patent 3,476,463, 4 November, 1969

3 Laser Beam Shaping, F.M. Dickey and S.C. Holswade, eds., Proc. SPIE 4095, 2000.

4 Laser Beam Shaping: Theory and Techniques, F.M. Dickey and S.C. Holswade, eds., Marcel Dekker, Inc., New York, 2000.

$5 \quad$ Laser Beam Shaping II, F.M. Dickey and S.C. Holswade, eds., Proc. SPIE 4443, 2001.

6 Chapter 1, pp. 12-14, Ref. 4.

7 D. L. Shealy, "Geometrical Methods," in Laser Beam Shaping: Theory and Techniques, pp. 163-213, Marcel Dekker, Inc., New York, 2000.

8 D. L. Shealy, "Theory of geometrical methods for design of laser beam shaping systems, in Laser Beam Shaping, F.M. Dickey and S.C. Holswade, eds., Proc. SPIE 4095, pp. 1-15, 2000.

9 J. H. McDermit and T. E. Horton, "Reflective optics for obtaining prescribed irradiative distributions from collimated sources," Appl. Opt. 13, pp. 1444-1450, 1974.

10 J. H. McDermit, "Curved reflective surfaces for obtaining prescribed irradiation distributions," Ph.D. Dissertation, University of Mississippi, Oxford, 1972. 
11 P. W. Malyak, "Two-mirror unobscured optical system for reshaping the irradiance distribution of a laser beam," Appl. Opt. 31, pp. 4377-4383, 1992.

12 D. L. Shealy and S. H. Chao, "Design and analysis of an elliptical Gaussian laser beam shaping system," in Laser Beam Shaping II, Fred M. Dickey, Scott C. Holswade, and David L. Shealy, eds., Proc. SPIE 4443, pp. 24-35, 2001.

13 P. W. Rhodes and D. L. Shealy, "Refractive optical systems for irradiance redistribution of collimated radiation: their design and analysis," Appl..Opt. 19, pp. 3545-3553, 1980.

14 P. W. Rhodes, "Design and analysis of refractive optical systems for irradiance redistribution of collimated radiation," M. S. Thesis, The University of Alabama in Birmingham, 1979.

15 J. A. Haffnagle and C. M. Jefferson, "Design and performance of a refractive optical system that converts Gaussian to a flattop beam," Appl. Opt. 19, pp. 5488-5499, 2000.

16 W. Jiang, D. L. Shealy, and J. C. Martin, "Design and testing of a refractive reshaping system," in Current Developments in Optical Design and Optical Engineering III,Robert E. Fisher and Warren J. Smith, eds. Proc. SPIE 2000, pp. 64-75, 1993.

17 W. Jiang, Application of a laser beam profile reshaper to enhance performance of holographic projection systems, Ph.D. Dissertation, The University of Alabama at Birmingham, 1993.

18 W. Jiang, D.L. Shealy, and K.M. Baker, "Optical design and testing of a holographic projection system," in Diffractive and Holographic Optics Technology, I Cindrich and S. H. Lee, eds., Proc. SPIE 2152, pp. 244-252, 1994.

19 N. C. Evans and D. L. Shealy, "Optimization-based techniques for laser shaping optics," in Laser Beam Shaping: Theory and Techniques, F. M. Dickey and S. C. Holswade, eds., Chapt. 5, Marcel Dekker, New York, 2000.

20 S. Solimeno, B. Crosignani, and P. DiPorto, Guiding, Diffraction, and Confinement of Optical Radiation, p. 49, Academic Press, Orlando, 1984.

21 A.K. Ghatak and K. Thyagarajan, Contemporary Optics, p. 24, Plenum Press, New York, 1980.

22 D.G. Koch, "Simplified Irradiance/Illuminance Calculations in Optical Systems," presented at the International Symposium on Optical Systems Design, Berlin, Germany, September 14, 1992, and published in Proc. SPIE 1780-14, 1992.

23 D.G. Burkhard, and D.L. Shealy, "Simplified formula for the illuminance in an optical system," Appl. Opt. 20, pp. 897-909, 1981.

24 D.G. Burkhard and D.L. Shealy, "A Different Approach to Lighting and Imaging: Formulas for Flux Density, Exact Lens and Mirror Equations and Caustic Surfaces in Terms of the Differential Geometry of Surfaces," in Materials and Optics for solar Energy Conversion and Advanced Lighting Technology, Carl. M. Lampert and Sandor Holly, eds., Proc. SPIE 692, pp. 248-272, 1986.

25 M. Born and E. Wolf, Principles of Optics, $5^{\text {th }}$ Edition, Pergamon Press, New York, 2000, p. 115.

26 Private communications with S-H. Chao.

27 D. F. Cornwell, "Non-projective transforms in optics," in New Methods for Optical, Quasi-Optical, Acoustic, and Electromagnetic Synthesis, W. Ross Stone, ed., Proc. SPIE 294, pp. 62-72, 1981.

28 W. Jiang and D.L. Shealy, "Development and testing of a refractive laser beam shaping system, in Laser Beam Shaping, Fred M. Dickey and S. C. Holswade, eds, Proc. SPIE 4095, pp. 165-175, 2000.

29 Focus Software, Inc., P.O. Box 18228, Tucson, AZ 85731.

30 C. Wang and D. L. Shealy, "Design of gradient-index lens system for laser beam reshaping," Appl. Opt. 32, pp. 4763-4769, 1993.

31 N. C. Evans and D. L. Shealy, "Design of a gradient-index beam shaping system via a genetic algorithm optimization method," in Laser Beam Shaping, F.M. Dickey and S.C. Holswade, eds., Proc. SPIE 4095, pp. 27-39, 2000.

32 N.C. Evans, "Genetic Algorithm Optimization Methods in Geometrical Optics," Ph.D. Dissertation, The University of Alabama at Birmingham, 1999.

33 UDG C1 is a CODE V variable that refers to "User-defined glass constant 1". C1 corresponds to a particular GRIN in the CODE V Lightpath catalog. The CODE V Lightpath GRIN glass catalog can be obtained from Optical Research Associates. 